\title{
Extension of Pinch Analysis to Targeting and Synthesis of Multi-Contaminant Material Recycle and Reuse Networks
}

\author{
Hon Huin Chin ${ }^{1}$, Peng Yen Liew ${ }^{2}$, Petar Varbanov ${ }^{1}$, and Jiří Jaromír Klemeš ${ }^{1}$ \\ ${ }^{1}$ Brno University of Technology Faculty of Mechanical Engineering \\ ${ }^{2}$ Universiti Teknologi Malaysia Malaysia-Japan International Institute of Technology
}

October 19, 2020

\begin{abstract}
A novel Pinch-based targeting and synthesis procedure in the domain of multiple constraints (e.g. multi-contaminants) for material recycle/reuse networks has been developed in this contribution. The main steps are the identification of the ranking order of material sinks/demands or sources/supplies. This is dependent on the limiting contaminants of sinks. Each contaminant/constraint is assigned a Pinch Diagram (Load vs Flowrate), and the Source and Sink Composite Curves (CC) are plotted for each diagram. The Source CC should be shifted until its lines could form a 'polygon' with the Sink CC, for which the points represent the vertices of the polygons. The sequential approach used is to first identify a preliminary resource target and allocated sources for each sink, then to follow certain heuristics for further reduction of the freshwater. The proposed approach provides a minimum resource target and a network design that achieves the targeted fresh resource along with graphical representation.
\end{abstract}

\begin{abstract}
An extended Pinch-based targeting and synthesis procedure in the domain of multiple constraints (e.g. multicontaminants) for material recycle/reuse networks has been developed in this contribution. The main steps are the identification of the ranking order of material sinks/demands or sources/supplies. This is dependent on the limiting contaminants of sinks. Each contaminant/constraint is assigned a Pinch Diagram (Load vs Flowrate), and the Source and Sink Composite Curves (CC) are plotted for each diagram. The Source CC should be shifted until its lines could form a 'polygon' with the Sink CC, for which the points represent the vertices of the polygons. The sequential approach used is to first identify a preliminary resource target and allocated sources for each sink, then to follow certain heuristics for further reduction of the freshwater. The proposed approach provides a minimum resource target and a network design that achieves the targeted fresh resource along with graphical representation.
\end{abstract}

Keywords : Process Integration; Multi-contaminant Water Pinch Analysis; Material Recycle/Reuse; Water Network Design

\section{Introduction}

To progress toward Circular Economy and to reduce the reliance on natural resources, the process industries have pursued material conservation as a key approach, which is mainly based on the concept of Process Integration [1]. El-Halwagi and Manousioutakhis [2] used the analogy with Heat Integration and introduced the problem of mass exchange network (MEN) for mass transfer operations in the process. Mass transfer processes include absorption, stripping, extraction, leaching, adsorption or ion exchange. They used the 
analogy to develop the related Composite Curves (CC) and Composition Interval Table (CIT) to determine the minimum water target, and the final network again is using the analogy from the Heat Grid Diagram [3] for design heuristics aided with Water Grid Diagram. Wang and Smith [4] again extended the Heat Pinch Approach to target the minimum freshwater consumption and wastewater discharged by the transfer of contaminants from process streams to water streams. A simplified design procedure was later introduced by Olesen and Polley [5], which results in better constructions for regeneration reuse and recycles designs. Hallale [6] introduced an extended targeting method for water minimisation. Gomes et al. [7] presented a heuristic algorithmic procedure, water source diagram (WSD), to synthesise water mass exchange networks. El-Halwagi and Manousiouthakis [8] showed that it is possible to target the minimum usage of external lean streams using again systematic representations passed on the physical understanding such as Composite Curves. El-Halwagi et al. [9] provided a single-stage targeting method to identify minimum resources for a single contaminant water network, with the solution strategy which identified through rigorous analysis. Both methods apply to mass exchange processes, and they rely on the basic principle of concentration driving force. For a review of the historical development of Water Pinch Analysis see, e.g. Foo [10]. Klemeš and Kravanja [11] provided an overview of the development of Process Integration prior to 2013, and Klemeš et al. [12] had more recently conducted a comprehensive overview of various extensions of PA in Mass Integration, including water and hydrogen integration.

However, a complicated issue is a solution in the case of multi-contaminant water flows. Various other strategies have been proposed to tackle this problem. Alva-Argaez et al. [13] developed Mixed-Integer Linear Programming (MILP) based multi-contaminant transhipment model used for targeting, particular for mass exchange networks and wastewater minimisation problems. Their model could tackle the general problem of mass exchange networks, but it could not evaluate the mixing effect of multi-contaminant problems. Gomes et al. [7] developed an algorithmic and graphical procedure, which is called Water Source Diagram (WSD) for a fixed load single contaminant system. This method is flexible and simple to use. It aims at minimising water consumption and provides the corresponding system network simultaneously directly. A series of works related to the use of WSD with multiple contaminants have been developed. An extension of the WSD for multiple contaminant systems was presented by Ulson de Souza et al. [14] to reduce freshwater consumption in an oil refinery. As the reference contaminant, they chose the one which was critical in the process, that is, the one which needs more water in operation. Karthick et al. [15] used the WSD with a mathematical method and generated a hybrid procedure to deal with the minimisation of freshwater for wastewater treatment. The WSD was used to provide starting points for the mathematical model. Gomes et al. [16] extended this method for maximum reuse and FL operations. This approach requires the choice of a reference contaminant and a reference operation to carry out the adjustments of concentrations in the required operations. Calixto et al. [17] developed a decomposition approach to be used with WSD, where the objective is to prevent the need of calculations to avoid violations in flowrate and/or concentration from the proposed network. This method also was applied to FL systems. Francisco et al. [18] extended this tool to fixed flowrate problem with multiple contaminants. The main steps involve the determination of the correct reference contaminants and operations, and later to adjust the inlet or outlet concentrations accordingly. Calixto et al. [19] provided an overview of the WSD and its application. Other attempts include the work of Castaño and Higuita [20], who used the property of turbidity (which sums a number of contaminants) in the design of water networks. The authors regarded turbidity as the key measured parameter and linear correlations of it were made with the concentration of the suspended solids. Mabitla and Majozi [21] presented a hybrid of graphical and mathematical approaches in solving multi-contaminant water and regeneration networks. The graphical approach involves the pre-processing steps to identify minimum water target and optimal regenerator removal ratios.

Another concept proposed by Liu et al. [22] is the concentration potential for a multi-contaminant problem. The concepts are presented based on the overall allocating possibility of source streams to demand streams. The concept is analogous to the single contaminant water network as it identifies the concentration order of the streams. Fan et al. [23] extended the concepts of concentration potential to the fixed flowrate operations. Li et al. [24] provide a review on this approach with their extension and applicability, and Zhao et al. [25] 
utilised the concept in designing heat-integrated water networks.

The Water Source Diagram (WSD) is a powerful tool and is widely used for industrial implementations due to its graphical visualisation platform [19]. Their algorithm to determine the resource target and network design require the adjustment on the concentration of the contaminant. In most of their works, they assumed the linear mass load ratio from Wang and Smith [26]. This might not be practical in realistic mass transfer operations. Calixto et al. [17] also explained that additional algorithm is needed to predict contaminant violation for the design framework. Francisco et al. [18] implemented this to the WSD algorithm, but the overall algorithm can be complicated. The potential concentration concept from Liu et al. [22] is another successful implementation, but it relies on the prioritisation of the sources. Chin et al. [27] mentioned that the prioritisation of the sources might not be the optimal allocation strategy, but to satisfy all the contaminant limits of the sinks as much as possible. This is applicable for fixed flowrate operations.

Using Mathematical Programming in MEN synthesis, Short et al.[28] formulated a comprehensive mixedinteger nonlinear program for MEN design. Oladosu et al. [29] presented an algebraic approach for simultaneous targeting and design of MENS with streams splitting. The proposed segregated Composition Interval Table (SECIT) was used to determine MEN Pinch Point and simultaneously target and design MENS. Yanwarizal et al. [30] adopted the Pinch based graphical tools and plotted the Composite Curves separating the streams instead of compositing the streams with similar concentration level. This strategy allows the simultaneous targeting and design of the MEN with a minimum fresh resource. However, their study was limited for single contaminant only.

In spite of the benefits of using MP in synthesising MEN network dealing with multi-contaminant problems, the approach is less favoured by design engineers due to the full automation of the solution. The superstructure formulation is demanding when the reasonable confidence in the proposed solutions and to produce solutions away from the optima. The solution strategy also provides restricted physical insights and analysis of the problem, making the internal bottlenecks unknown. This is true; especially when an authentic problem involves a lot of contaminants or streams properties constraints. The development of PA is an essential step for industrial application because it reveals the inherent system limitations - targets of the resource supply and the internal bottlenecks. These targets are then used in the detailed design model setting the optimisation strategy and providing bounds on the key variables. This is the logic which has led to the Process Integration strategy to identify the target for minimum usage of fresh resources ahead of detailed design, e.g. Heat Integration [3].

Based on the analysis, the objective of this paper is to develop a Pinch-Based simultaneous targeting and design of minimum fresh resource using interactive graphical representations. The specific problem of water using systems with multiple contaminants is studied in this paper. The proposed method involves the ranking of different resources to be reuse/ recycling according to its composition of various contaminants as well as assigning sinks to the proper contaminant cascades. The graphical plots using the load vs flowrate diagram can show the Pinch Point for different contaminants, maximum mass recovery, source allocation and minimum external resource targets for individual sinks simultaneously. The plot result can then be translated into source allocation network with the optimal design without the need to perform calculations to check for mass transfer feasibility.

\section{Concepts development}

This section first presents the necessary concepts used to perform graphical targeting and design of the multicontaminant material network. Section 2.1 explains the proposed 'Polygon' rule with graphical visualisation. Section 2.2 describes the assignment of the sinks to proper contaminant cascade. Section 2.3 compares the source allocation pattern between the single contaminant and multiple contaminants. Section 2.4 then describes the network design framework for each sink using graphical method.

The typical Water Pinch Analysis (PA) could be performed to target freshwater demand. There are various 
versions of graphical tools dealing with material recycle/reuse problems. For fixed load operation, one of the popular tools is the Water Surplus Diagram [6], which shows the plot of impurity concentration vs flowrate. The plot is useful to determine the fresh resource target only if no mixing between lower and higher quality sources, relative to the sink is allowed. This added benefit cannot be shown by the plot. If it is to be used, an initial guess of a freshwater target is required. For fixed flowrate operation, El-Halwagi et al. [9] proposed the load vs flowrate diagram as an alternative to determining the freshwater target. The strength of this plot is it accounts for the mixing of sources. This targeting tool is also non-iterative and systematic in nature, providing a simpler alternative for graphical targeting purpose. In this work, the load vs flowrate used is used to solve the targeting problem with multi-contaminants material recycle/reuse.

\section{Polygon rule for sources mixing}

Yang et al. [31] presented a triangle rule in the graphical method for hydrogen Pinch Analysis, accounting for the purification process. In their work, they proposed the purification of the hydrogen feed stream into purified products and tail gas. The mass of the contaminants is conserved around the purification unit. Figure 1a shows the presented 'polygon' (triangle) rule. The feed stream that has certain flowrate and quality load is plotted on a load vs flowrate diagram. The horizontal distance of line AC represents the flowrate of the feed stream, while the vertical distance is the contaminant load of the stream. The gradient of the line represents the contaminant concentration of the feed stream. The feed stream is balanced by lines 'AB' and 'BC', which represent the load and flowrate contributions of the purified product and the tail gas. This concept can be applied to streams mixing as well, but the process direction is reverse. A dirtier stream (line 'AB') can be mixed with cleaner stream (line 'BC') to form a stream 'AC'. It doesn't matter which path to go from point 'A' to point ' $\mathrm{C}$ ', as long as the final point ' $\mathrm{C}$ ' is reached. The direction of the process has to be denoted separately, as the diagram represents only the balance.

The 'triangle' rule can be generalised into a 'Polygon' rule, as presented in Figure 1b. The feed stream AC can be balanced by more than two streams such as $\mathrm{AB}, \mathrm{BD}, \mathrm{DE}$ and $\mathrm{EC}$.

(a)

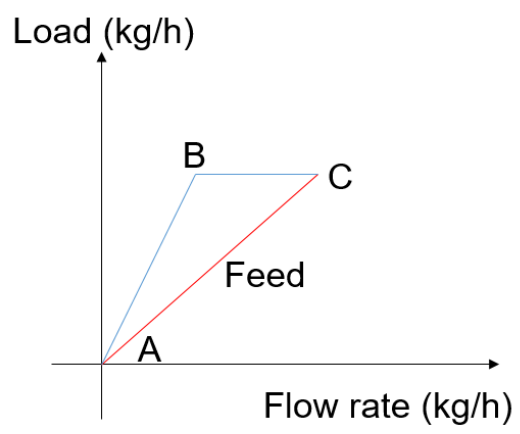

(b)

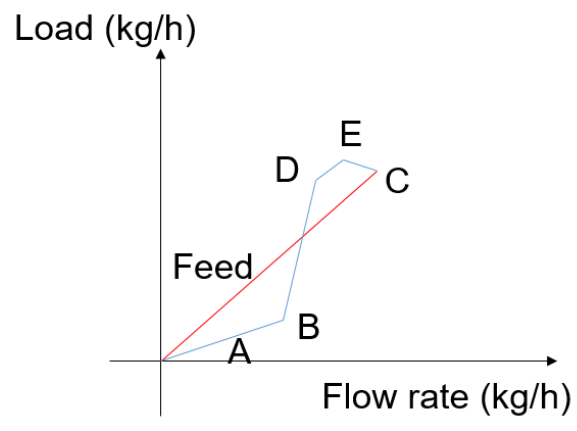

Figure 1: Demonstration of 'polygon' rule, adapted from Yang et al. [31]

The concept of the 'Polygon' rule can be applied to the streams mixing as well. Considering a mass balance of sinks that are fulfilled by various sources (for a single contaminant)- Appendix A: Eqs(A3-A4):

$$
\begin{array}{llll}
\hline F_{\mathrm{SKj}}=\sum_{i} F_{S R i, S K j}+F_{F w, S K j} & \forall j & (1) \\
\hline \sum_{i} F_{S R i, S K j} C_{\mathrm{SRi}}+F_{F w, S K j} C_{\mathrm{Fw}} \leq F_{\mathrm{SKj}} Z_{\mathrm{SKj}} & \forall j \quad \forall k & (2) \\
\hline
\end{array}
$$

Where $\mathrm{F}_{\mathrm{Fw}, \mathrm{SKj}}$ is freshwater to sink ' $\mathrm{j}$ ' flowrate, $\mathrm{F}_{\mathrm{SRi}, \mathrm{SKj}}$ is source ' $\mathrm{i}$ ' to sink ' $\mathrm{j}$ ' flowrate, $\mathrm{F}_{\mathrm{SKj}}$ is the sink ' $\mathrm{j}$ ' 
flowrate, $\mathrm{C}_{\mathrm{SRi}}$ is the concentration of contaminant in source ' $\mathrm{i}$ ', $\mathrm{C}_{\mathrm{Fw}}$ is the concentration of contaminant in freshwater, and $\mathrm{Z}_{\mathrm{SKj}}$ is the concentration of contaminant in sink ' $\mathrm{j}$ '.

Figure 2a-b show examples of feasible source mixing. As long as the final cumulative load of the source Composite Curve (CC) touches or is below the endpoint (final cumulative load) of the sink CC, the maximum quality limit of the sink is still fulfilled (Eq 2). This is in-line with the concept of stream mixing. A dirtier source (higher gradient line) can be mixed with a cleaner source (lower gradient line) to fulfil the concentration or quality limit of the sink demand- Figure 2a. The cumulative load of sources is located below a load of sink CC means that the total allocated source streams have a lower concentration than the maximum limit of sink's concentration- Figure 2b. Figure 2c is the example of infeasible source mixing as the cumulative load of the sources is above the sink's requirement. In this scenario, more freshwater is needed by shifting the source CC to the right.

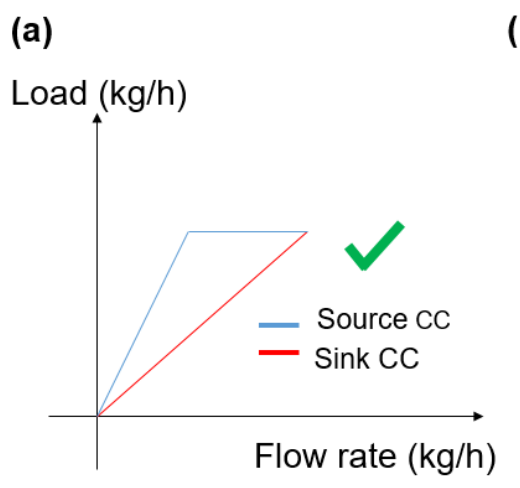

(b)

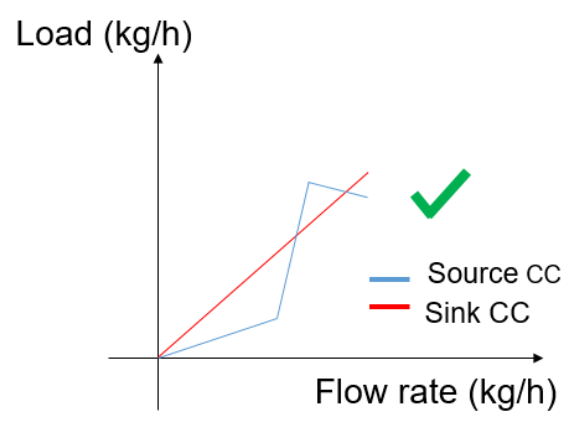

(C)

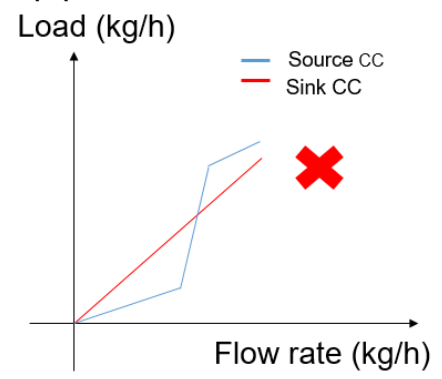

(d)

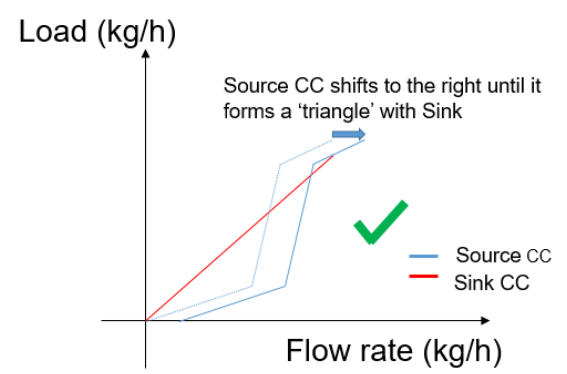

Figure 2: Different sources of mixing scenarios

1.

\section{Sink classification}

Classification of sinks to contaminant cascades

Chin et al. [27] proposed an indicator to identify the sinks' contaminant cascades and limiting contaminants. For a problem with 'k' contaminants with ' $N$ ' internal sources, assuming a single sink (SK1), the real freshwater target is shown in Eq (3) - see the derivation in Appendix A- Eq (A13). To identify the preferred source ranking for the specific sink, the ratio of sink concentration to the source concentration play an important role. It has been proposed that the largest source concentration of contaminant ' $\mathrm{k}$ ' $\left(\mathrm{C}_{\mathrm{k}, \mathrm{SR} \_ \text {max }}\right)$ 
is used because it also shows exactly the prioritisation of sources if more than two sources are used. For

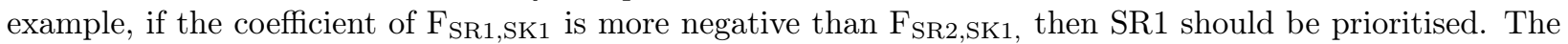
maximum source concentration can also be treated as a normalisation constant.

$F_{F w, S K 1} \geq\left(F_{S R 1, S K 1}\left[\frac{C_{k, S R 1}-C_{k, F W}}{C_{k, S R_{-} \max }-C_{k, F W}}-1\right]+\ldots+F_{S R N, S K 1}\left[\frac{C_{k, S R N}-C_{k, F W}}{C_{k, S R_{-} \max }-C_{k, F W}}-1\right]+F_{S K 1}\left[1-\left(\frac{Z_{k, S K 1}-C_{k, F W}}{C_{k, S R_{-} \max }-C_{k, F W}}\right)\right.\right.$

The proposed ratio $\left(\mathrm{Z}_{\mathrm{k}, \mathrm{sk}} / \mathrm{C}_{\mathrm{k}, \mathrm{SR} \_ \text {max }}\right)$, where $\mathrm{Z}_{\mathrm{k}, \mathrm{SKj}}=\mathrm{Z}_{\mathrm{k}, \mathrm{SKj}}-\mathrm{C}_{\mathrm{k}, \mathrm{FW}}$ and $\mathrm{C}_{\mathrm{k}, \mathrm{SR} \_ \text {max }}=\mathrm{C}_{\mathrm{k}, \mathrm{SR} \_ \text {max }}$ $\mathrm{C}_{\mathrm{k}, \mathrm{FW}}$. helps to identify the source prioritisation as this ratio constitutes the constant term in expression (3). If the ratio is smallest for contaminant ' $\mathrm{k}$ ', then the constant term: $F_{S K 1}\left[1-\left(\frac{Z_{k, S K 1}-C_{k, F W}}{C_{k, S R_{-}} \max -C_{k, F W}}\right)\right]$ has the largest value which means the source allocation is more likely to follow the prioritisation sequence for contaminant 'k'. The optimal source allocation should be done to minimise this term. However, this does not mean that the sink should follow exactly the source prioritisation sequence for contaminant ' $k$ ', because different sources are traded-off by other contaminants. The proposed concentration ratio shows the limiting contaminant for a specific sink, and also show which source prioritisation order is likely to be followed.

Classification of sinks to Below or Above Pinch Regions

Based on the definition of Material Pinch Point, the material streams located below the Pinch Region require a fresh resource, while the one located above the Pinch Region does not require fresh resource and the unused sources are discharged. In other words, the Pinch Point separates the material sinks into two parts [27]:

- Above the Pinch Point, the sinks do not require freshwater, i.e. the total freshwater target, $\mathrm{F}_{\mathrm{FWT}}=0$.

- Below the Pinch Point, the sinks require freshwater, i.e. the total freshwater target, $\mathrm{F}_{\mathrm{FWT}}>0$.

For a multi-contaminant case, it is imperative to determine the prior classification of the sinks to Below or Above the Pinch Regions in each contaminant cascade. This is because the sequence of sink is needed to be determined so that the allocation of source is performed. The existence of multiple contaminants makes the identification difficult. To address this problem, the individual concentration ratio of the source to the sink $\left(\mathrm{C}_{\mathrm{SR}} / \mathrm{Z}_{\mathrm{SK}}\right)$ plays an important role. The following heuristic for classification of sinks to above or below Pinch Region is used. For detailed explanation please refer to Chin et al. [27].

1. Determine the ratio of shifted sink concentration $\left(\mathrm{Z}_{\mathrm{k}, \mathrm{SKj}}=\mathrm{Z}_{\mathrm{k}, \mathrm{SKj}}-\mathrm{C}_{\mathrm{k}, \mathrm{FW}}\right)$ to the shifted source concentration $\left(\mathrm{C}_{\mathrm{k}, \mathrm{SRi}}=\mathrm{C}_{\mathrm{k}, \mathrm{SRi}}-\mathrm{C}_{\mathrm{k}, \mathrm{FW}}\right)$, i.e. $\mathrm{Z}_{\mathrm{k}, \mathrm{sk}}^{*} / \mathrm{C}^{*}{ }_{\mathrm{k}, \mathrm{SRi}}$ for each sink-source pair and for each contaminant ' $k$ ', Where and

2. For each sink 'j',

1. Identify the smallest value of the concentration ratio of sink 'j' with all the sources.

2. Identify which pairs have ratio $\geq 1$ and which have $<1$.

3. Calculate the number of sources $\left(\mathrm{N}_{\mathrm{SR}+}\right)$ that contribute to the ratio $\geq 1$ and the number of sources $\left(\mathrm{N}_{\mathrm{SR}-}\right)$ that contribute to ratio $<1$.

4. If the total flowrate of $\mathrm{N}_{\mathrm{SR}+} \geq$ total flowrate of $\mathrm{N}_{\mathrm{SR}}$, then sink ' $\mathrm{j}$ ' is classified as a sink that does not require fresh resource. Else, sink ' $\mathrm{j}$ ' is classified as a sink that requires fresh resource.

5 .

\section{Source allocation pattern for multi-contaminants}

In the domain of single contaminant problems, the water sources are prioritised with the ascending order of concentration. This strategy is well-proven in the paper of El-Halwagi et al. [9], which means cleaner (higher quality) sources should be fully used up first. However, the source prioritisation becomes complicated when multiple contaminants are involved. The following sub-sections explain the source allocation characteristics 
for a multi-contaminant problem, and to infer the source allocation steps to be followed, to achieve the minimum freshwater target.

Consider the two-contaminant example presented in Table 1 below, and Example 1a features a problem with conflicting sources. If the only contaminant 'A' is considered, SR1 is cleaner than SR2, while for contaminant 'B', SR2 is cleaner than SR1. The prioritisation sequence of the sources is not obvious in this case. Example $1 \mathrm{~b}$ features a problem with non-conflicting sources. It is apparent that SR1 and SR2 are not conflicting in

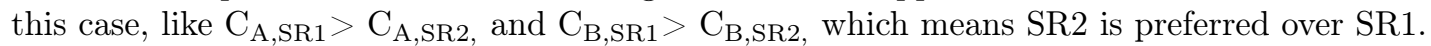

Table 1: A two-contaminant problem with both examples of conflicting and non-conflicting sources

\begin{tabular}{lllll}
\hline SR & $\mathrm{F}_{\mathrm{sr}}(\mathrm{t} / \mathrm{h})$ & Example 1a: Conflicting sources & Example 1a: Conflicting sources & Example 1b: Non-conflicting sourc \\
\hline & & $\mathbf{C}_{\mathbf{A}}, \mathbf{s r}(\mathbf{p p m})$ & $\mathbf{C}_{\mathbf{B}}, \mathbf{s r}(\mathbf{p p m})$ & $\mathbf{C}_{\mathbf{A}}, \mathbf{s r}(\mathbf{p p m})$ \\
1 & 23 & 500 & 30 & 500 \\
2 & 123 & 50 & 200 & 50 \\
$\mathrm{Fw}$ & - & 0 & 0 & 0 \\
$\mathrm{SK}$ & $\mathbf{F}_{\mathbf{s k}}(\mathbf{t} / \mathbf{h})$ & $\mathbf{Z}_{\mathbf{A}}, \mathbf{s k}(\mathbf{p p m})$ & $\mathbf{Z}_{\mathrm{B}}, \mathbf{s k}(\mathbf{p p m})$ & $\mathbf{Z}_{\mathbf{A}}, \mathbf{s k}(\mathbf{p p m})$ \\
1 & 50 & 100 & 100 & 100 \\
\hline
\end{tabular}

Figure 3a shows the plot of the relationship (Example 1a) between the freshwater target for SK1 and the flowrate of SR1 supplied to SK1, for both contaminants. Note that the flowrate of SR2 (F $\left.\mathrm{F}_{\mathrm{SR} 2, \mathrm{SK} 1}\right)$ is dependent on SR1 and $\mathrm{Fw}$, so its flowrate can be computed by performing mass balance around SK1, which is $\mathrm{F}_{\mathrm{SR} 2, \mathrm{SK} 1}=\mathrm{F}_{\mathrm{SK} 1}-\mathrm{F}_{\mathrm{FW}, \mathrm{SK} 1}-\mathrm{F}_{\mathrm{SR} 1, \mathrm{SK} 1}$.

The shaded region represents the feasible region of the freshwater requirement. By observing Figure 3a, it is apparent that the minimum freshwater always falls into the boundaries of the feasible region, i.e. one of the impurity constraints would always be active. The interesting point is where both the boundary lines intercept, which represents both impurity constraints are active. As SR1 and SR2 are conflicting sources in both contaminants 'A' and 'B', the boundary lines are linear with the respective negative and positive gradient. Assuming no limitation on SR1 and SR2 flowrates, the minimum freshwater point is always at the point where all impurity constraints are active. If one of the sources has limited flowrate, the minimum point cannot be achieved. In such a case, any points along the lowest boundary line are the minima. Figure $3 \mathrm{~b}$ shows the plot for non-conflicting sources (Example 1b). In this case, the intersection point between the boundary lines is no longer the minimum point, because both lines are with a positive gradient. The usage of SR1 should be minimised, to reduce the freshwater target, which means SR2 should be maximised. SR2 should be fully used up first before SR1 is used. The source prioritisation becomes similar to the single contaminant case.
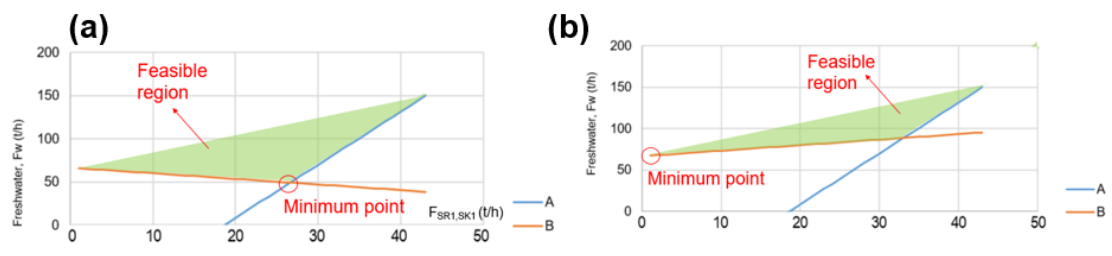

Figure 3: Freshwater flowrate vs flowrate of SR1 (a) Conflicting SR1 and SR2 (b) Non-conflicting SR1 and SR2

The source allocation pattern becomes slightly more complicated if more than two contaminants are involved. As established in the two-contaminant problem, the interception point where all impurity constraints are active represents the minimum freshwater point. This is similar to any problem with 'k' contaminants [27]. 
Based on the geometric property of straight lines, if there exists a common intersection point among all ' $k$ ' straight lines, then no other intersection points exist. The converse of the property is also true, i.e. If the intersection point for lines of contaminant pairs A-B, lines B-C/A-C are the same, then there exists a common intersection point [27]. This property is important as it determines whether a point exists where all impurity constraints are active. If such a point exists, then it represents the minimum freshwater point (if all the sources have enough flowrate). The common point is the minimum freshwater point, only if there is at least one conflicting sequence of the two sources. If there are no sources which have a conflicting sequence, the common point is not the minimum point.

However, not every source allocation problem has a common intersection point. To achieve optimum recycle with minimum fresh resource, the concentration or load limits for all contaminants for all sinks should be reached (if possible), or at least several limits are reached- see Chin et al. [27]. This complicates the problem as the source arrangement is not based on the concentration order any more. It becomes tedious if ones have to determine the source allocation amount manually. Section 2.4 is devoted to the explanation of the proposed heuristics to determine the number of sources to be allocated.

\section{Network design: source allocation through the graphical plot}

An efficient graphical targeting or design method for single contaminant material recycle/reuse problem is the cumulative load vs cumulative flowrate diagram [9]. This strategy is equivalent to sequential, fulfilling each sink with internal resources, which are prioritised with contaminant concentrations. Each segment of the line represents each sink/source, with a horizontal length of the line represents the flowrate and vertical length represents the contaminant load. The figures are usually plotted by compositing each sink/source line based on the ascending order of the contaminants' concentration.

In the case of single contaminant, the recycle strategy first starts with the cleanest (highest quality) sink with the use of the cleanest source. The source line is moved horizontally (pure fresh resource) until it touches the sink line, and the source is located below the sink line - see Figure 4. The overlapped flow rate represents how many sources can be used for the sink. The remaining flow of the sink can only be fulfilled by fresh resource. The remaining flowrate of the source is transferred to the next sink - Figure 4. For the third sink, it can be fully fulfilled by the mix of the remainder of the second source and third source, without the use of fresh resource - Figure 4. Adding together the fresh resource flow, Figure 4 shows the typical representation of the Composite Curves in Load vs Flow diagram. The total freshwater target is so $\left(\mathrm{F}_{1}+\mathrm{F}_{2}\right)$, which is the fresh resource requirement.

In fact, the water network design with source allocation can already be determined from Figures 4a-c. For example, Figure 4a shows only part of source 1 can be allocated to sink 1 . Figure $4 \mathrm{~b}$ shows the allocation of sources 1 and 2 to sink 2 . The benefits of determining the freshwater target for each sink sequentially allows for that sink the source allocation to be determined simultaneously.

If a similar strategy is applied in the multi-contaminant problem, slight adjustment on the Source CC has to be done. For illustrative purpose, Figure 5 shows that for the first sink, the limit for contaminant A is reached. There is still room for contaminant B for the first sink as its limit is 


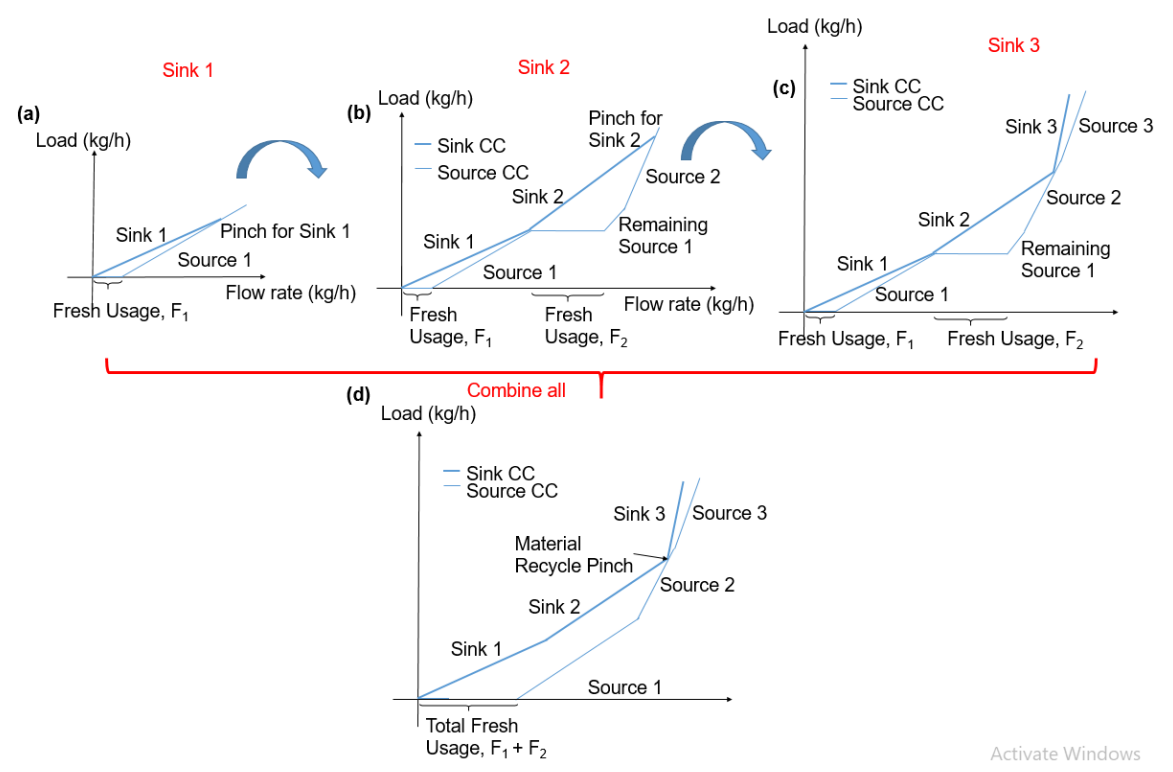

Figure 4: Sequential source allocation with Pinch-based methodology with graphical representation, adapted from El-Halwagi et al. [9]

not reached (a triangle is formed). This also means the limiting contaminant for the first sink is A (which is dependent on the source sequence). Moving on to the second sink, the starting point for the source CC (for contaminant B) after the first sink should be shifted vertically upwards to account for the unfulfilled concentration limit for the first sink - Figure 5b. Note that the 'triangle' formed in Figure 5a around Sink CC (contaminant B) is still feasible, as explained in section 2.1. For the second sink, the limit for contaminant B is reached, so the source CC for contaminant A after the second sink should be shifted vertically downwards when the remaining is transferred to the third sink - Figure $5 \mathrm{c}$. 
(a)

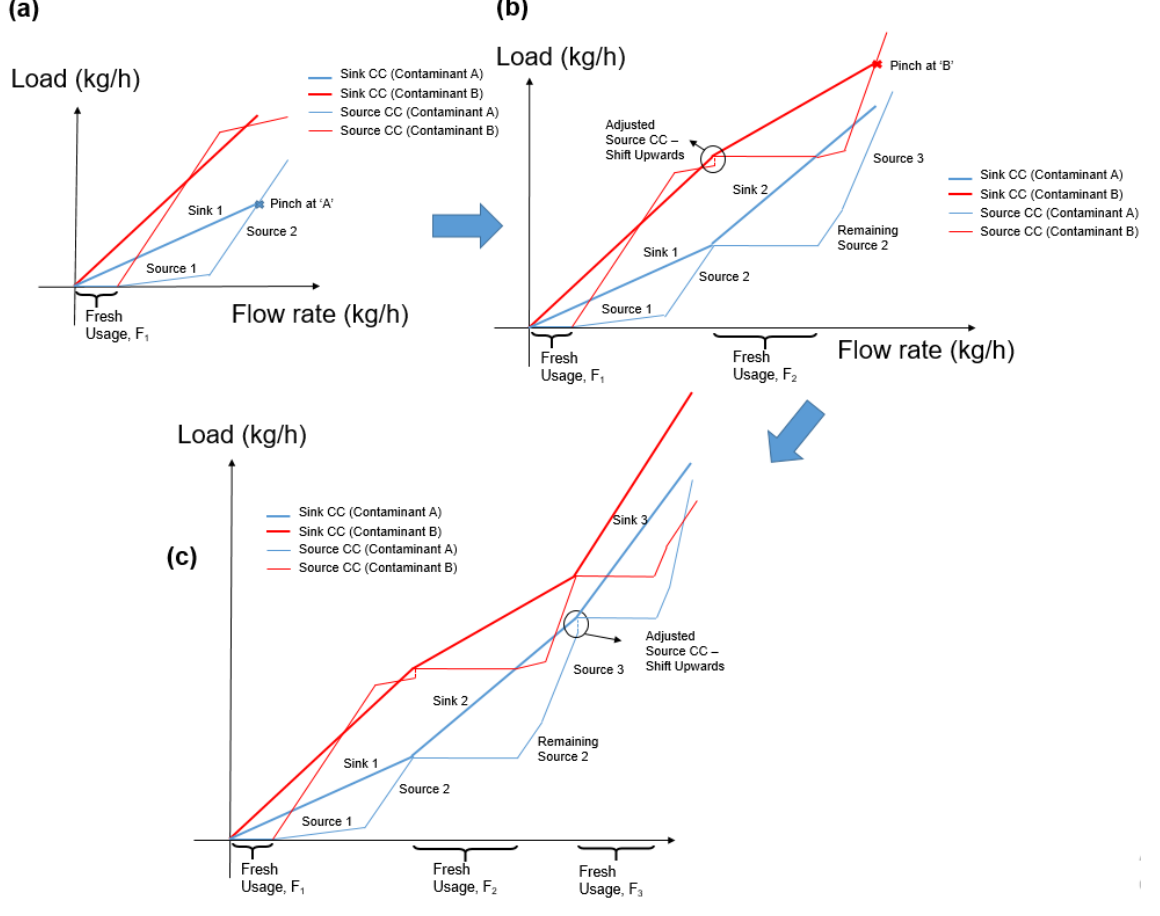

Figure 5: Sequential source allocation with a developed methodology for multiple contaminants

However, the optimal source allocation strategy in a multi-contaminant water network is not straightforward. The prioritisation of the cleaner source is no longer applicable for the multi-contaminant case as the ranking of the sources is not obvious. This is demonstrated in Figure 6.

Figure 6a shows the demonstration plot of the sink and source CC for a single sink and two sources. Source 1 and Source 2 are conflicting. The sources are arranged based on the ascending order of contaminant 'A' concentration. In this example, the Pinch point occurs at the contaminant 'B' if Source 1 is prioritised over Source 2, while for contaminant 'A' Pinch is not reached. However, there is room for freshwater reduction by reducing Source 1 flowrate allocated to sink 1. This is because Source 1 has a higher concentration in Contaminant B than Source 2. Reducing the use of Source 1 helps to reduce the load for Contaminant B. As presented in Section 2.3 for the case of conflicting sources, the optimal freshwater requirement occurs when all contaminant limits are reached. To achieve this, Source 1 can be reduced until the distances from the endpoint of both sink CCs to both of the source CCs are identical (see Figure 6b), i.e. Pinch Points occur for all the contaminants. The source CCs can be shifted to the left until both Pinch points are reached (Figure $6 c$ ). This is the optimal source allocation strategy for the specific sink. Similar steps can be repeated for the subsequent sinks.

The determination of the sources flowrates by manually adjusting the curves can be time-consuming, especially when there are plenty of sources available. This issue can be solved by using Eqs(4-5). To determine the reduced flowrates of the sources, one can set the following condition from $\mathrm{Eq}(4)$ : 
(a)

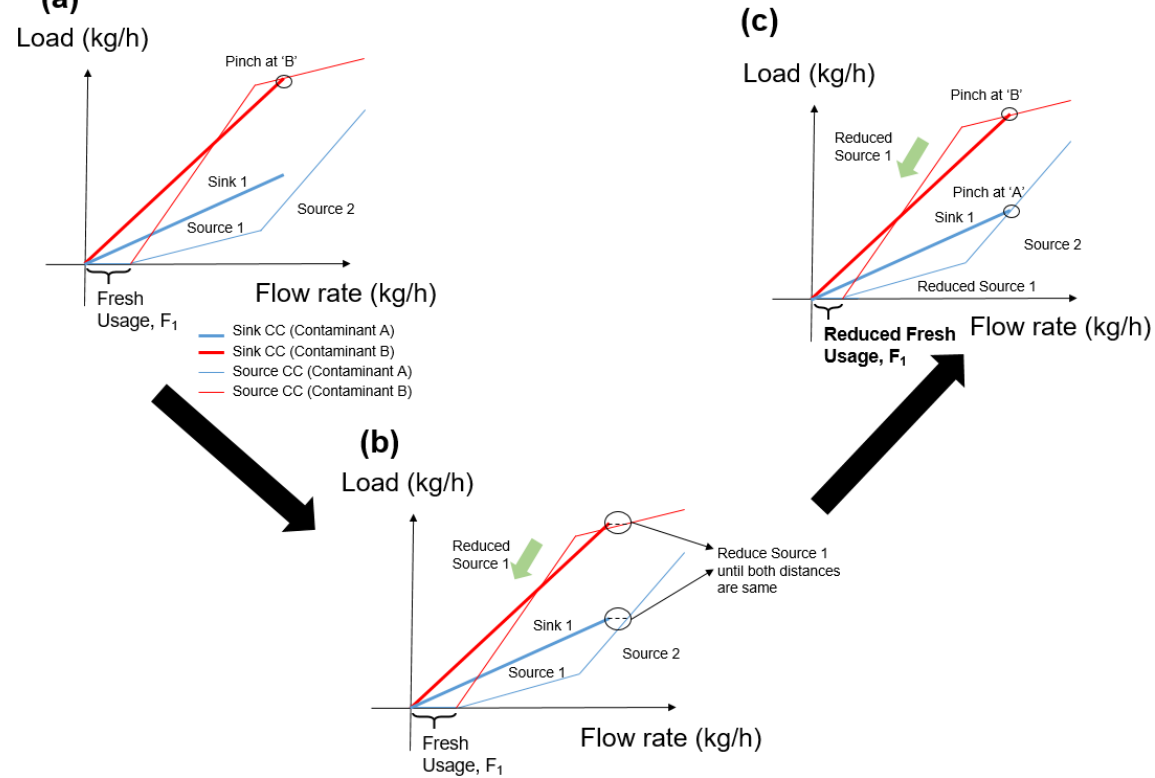

Figure 6: Source allocation strategy for a single sink

\begin{tabular}{ll}
\hline$F_{F w, S K 1}$ for contaminant $A=F_{F w, S K 1}$ for contaminant B & $(4)$ \\
\hline$F_{F w, S K 1}=\sum_{i=1}^{I} F_{S R i, S K j}\left[\frac{\left(C_{k, S R i}-C_{k, F w}\right)}{\left(C_{k, S R i_{s}}-C_{k, F w}\right)}-1\right]+F_{\mathrm{SKj}}\left[1-\frac{\left(Z_{k, S K j}-C_{k, F w}\right)}{\left(C_{k, S R i_{s}}-C_{k, F w}\right)}\right]$ & $(5)$ \\
\hline
\end{tabular}

Where $C_{k, S R}$ ref is the concentration of a reference source. In the example above, the reference source should be source 2 when the flowrate for source 1 is to be determined. Otherwise, if the flowrate of source 2 should be determined, then source 1 should be used as the reference source.

The freshwater reduction by reducing the flowrates of the sources is only possible when the Pinch Point occurs at the non-limiting contaminants. For example, if the Pinch Point occurs at the contaminant 'A' after shifting the source CC in Figure 5a, the freshwater requirement is already at the optimum level. There is no room for further reduction. The ratio with the sink's concentration with the maximum source concentration ratio can be used as the indicator to determine the limiting contaminant for the specific sink (see Section 2.2.1).

\section{Overall graphical design procedure}

For a more general ' $k$ ' contaminants case and many sources available. The ideal case of source allocation depends on the number of contaminants. For 2 contaminants, it should be paired with 2 sources (3 equations: Sink mass balance and both impurity constraints with 3 unknowns: $F_{F_{w}}, F_{\mathrm{SR} 1}, \mathrm{~F}_{\mathrm{SR} 2}$ ). For 'k' contaminants, then pair with ' $k$ ' sources ('k' equations with ' $k$ ' unknowns). The following Source Allocation Step is presented to identify the optimal source flowrates for a specific sink:

1. For a specific sink, check whether the Pinch Point occurs at the CCs that correspond to the main limiting contaminant. The main limiting contaminant is the one producing the smallest ratio of sink's concentration to the maximum source concentration (see Section 2.2.1). If the Pinch is at the limiting contaminant, go to step (vi), else continue to step (ii). 
2. For a sink, if there are 'k' contaminants, then pair with 'k' sources that are arranged in the order based on the contaminant cascade that the sink is assigned to.

For example, if SK1 is assigned to contaminant cascade 'A', then pair with the first ' $k$ ' sources that are arranged with ascending order of concentration 'A'. This is explained in the next point.

Set $F_{F w, S K 1}$ for contaminant ' $\mathrm{k} 1$ ' $=F_{F w, S K 1}$ for contaminant 'k2' for each binary contaminant pairs minus $1\left({ }^{\mathrm{k}} \mathrm{C}_{2}-1\right)$. Determine the flowrates of the first ' $\mathrm{k}$ ' sources using $\mathrm{Eq}(3)$ by using one of the sources as a reference source.

- For example, if there are 3 contaminants, i.e. $\mathrm{k}=\{\mathrm{A}, \mathrm{B}, \mathrm{C}\}$, then set a condition $F_{F w, S K 1}=F_{F w, S K 1}$ for each binary pairs, i.e. A-B, B-C, A-C. By setting $F_{F w, S K 1}$ for $\mathrm{A}=F_{F w, S K 1}$ for B and $F_{F w, S K 1}$ for $\mathrm{B}=F_{F w, S K 1}$ for $\mathrm{C}$, this means the freshwater requirement for $\mathrm{A}$ is set to equal to for $\mathrm{C}$ as well. Setting the freshwater conditions is sufficient for 2 of the pairs $\left({ }^{3} \mathrm{C}_{2}-1\right)$.

- For 3 contaminants case, solve the system of equations with $\mathrm{Eq}(5)$ for both A-B and B-C pairs. If SR1, SR2 and SR3 are the first 3 sources in the cascade, determine the flowrate of SR1 and SR2 by setting SR3 as a reference source. This is to achieve the limits for 3 contaminants. As there are 3 equations ( 1 sink mass balance equation and 2 Eqs(5) for two of the contaminant pairs) for 3 contaminants, to ensure zero degrees of freedom, 3 sources are needed.

- Repeat this by setting each source as the reference source until all the flowrates have been identified.

1. If one of the source flowrates is determined as negative, this means the scenario where all contaminants have Pinch Points does not exist. If this is the case, repeat the procedures (i) to (iv) for 'k-1' contaminants. If not, go to step (vi)

2. Check if the sources flowrates are enough to cover the sink. If one of the sources has not enough flowrates, fully use up the source. Repeat the procedures (i) to (iv) with the next 'k' sources.

For example, for the 3 contaminants example, if the optimal flowrates of SR1 exceed the available SR1, fully use up SR1 for the sink. Repeat the similar procedures using the next three sources, i.e. SR2, SR3 and SR4.

Repeat procedures (i) to (v) until all sinks are fulfilled

Based on these observations, the following overall design procedures using graphical methodology with Pinch concept are proposed:

1. Assign the sink to proper contaminant cascade. This can be done by determining the ratio of sink's concentration to the maximum source concentration (see Section 2.1)

2. For each sink, determine whether it should be Below or Above the Pinch.

3. Start the design procedures with the contaminant cascade that contains the sink that has the highest flowrate.

4. Arrange the sink in the contaminant cascade with ascending order of the concentration of contaminant based on the cascade it has been assigned to.

5. Perform the Source Allocation Steps for the sinks that are Below the Pinch.

6. Repeat the procedures (iii) to (v) until the sinks (Below the Pinch) for the next contaminant cascade.

7. Allocate the remaining sources to the sinks that are Above the Pinch.

\section{Case studies}

Several case studies are used to elucidate the applicability of the proposed methodology. The first case study is a two-contaminant problem, with four sources and four sinks available based on Teles et al. [32]. Different scenarios are explored, which includes changing the sequence of the sources and the existence of a sink that does not require water (above the Pinch Region). The second case study involves a three-contaminant problem in a pulp and paper mill from Lovelady et al. [33] - see Appendix B. The third case involves a 
larger case study in an India starch industry, with data retrieved from Dakwala et al. [34] - see Appendix C.

Case study 1: Two-contaminant problem

This case study is an illustrative example with two contaminants from Teles et al. (2008). The data is manipulated to explore different scenarios that involve changing the concentration or flowrate of sources.

\section{Scenario 1: Complete conflicting sources}

The first scenario explores a case with complete conflicting sources sequence, i.e. the source prioritisation for contaminant ' $\mathrm{A}$ ' is completely opposite with contaminant ' $\mathrm{B}$ '. The data is presented in Table 2 :

Table 2: Data for scenario 1 of case study 1

\begin{tabular}{llll}
\hline $\mathrm{SR}$ & $\mathrm{F}_{\mathrm{sr}}(\mathrm{kg} / \mathrm{h})$ & $\mathrm{C}_{\mathrm{A}, \mathrm{SR}}(\mathrm{ppm})$ & $\mathrm{C}_{\mathrm{B}, \mathrm{SR}}(\mathrm{ppm})$ \\
\hline 1 & 23 & 50 & 300 \\
2 & 47 & 100 & 120 \\
3 & 123 & 150 & 100 \\
4 & 60 & 250 & 80 \\
$\mathrm{Fw}$ & - & 0 & 0 \\
$\mathrm{SK}$ & $\mathbf{F}_{\text {sk }}(\mathbf{k g} / \mathbf{h})$ & $\mathbf{Z}_{\mathbf{A}, \mathbf{S k}}(\mathbf{p p m})$ & $\mathbf{Z}_{\mathrm{B}, \mathbf{S K}}(\mathbf{p p m})$ \\
1 & 23 & 20 & 60 \\
2 & 47 & 50 & 20 \\
3 & 123 & 100 & 150 \\
4 & 70 & 200 & 80 \\
\hline
\end{tabular}

The first step is to identify which contaminant cascades for each sink should be assigned. The purpose of this step is also to determine the most likely source prioritisation sequence for each sink. This is performed by determining the ratio of shifted sink concentration to the shifted maximum of source concentration $\left(\mathrm{Z}_{\mathrm{k}, \mathrm{sk}} / \mathrm{C}^{*}{ }_{\mathrm{k}, \mathrm{sr} \_ \text {max }}\right)$ for each contaminant ' $\mathrm{k}$ '. Table 3 presents the results, and it shows that SK1 and SK3 should be in contaminant cascade 'A', while SK2 and SK4 should be in contaminant 'B' cascade, based on the smallest value of the ratio.

Table 3: Identification of source prioritisation sequence/contaminant cascade of each sinks for Case study 1 scenario 1

\begin{tabular}{lllll}
\hline & $\mathrm{Z}_{\mathrm{A}, \mathrm{SK}} / \mathrm{C}_{\mathrm{A}, \mathrm{SRmax}}$ & $\mathrm{Z}_{\mathrm{B}, \mathrm{SK}} / \mathrm{C}_{\mathrm{B}, \mathrm{SRmax}}$ & & \\
& $\mathrm{C}_{\mathrm{A}, \mathrm{SRmax}}=250$ & $\mathrm{C}_{\mathrm{B}, \mathrm{SRmax}}=300$ & Minimum ratio & Cascade \\
\hline SK1 & $\mathbf{0 . 0 8}$ & 0.20 & 0.08 & $\mathrm{~A}$ \\
SK2 & 0.20 & $\mathbf{0 . 0 7}$ & 0.07 & $\mathrm{~B}$ \\
SK3 & $\mathbf{0 . 4 0}$ & 0.50 & 0.40 & $\mathrm{~A}$ \\
SK4 & 0.8 & $\mathbf{0 . 2 7}$ & 0.27 & $\mathrm{~B}$ \\
\hline
\end{tabular}

The second step is to classify the sinks into Above and Below Pinch regions, for both contaminants 'A' and 'B'. The source order for contaminant 'A' is: SR1->SR2->SR3-SR4, while the source order for 'B' is: SR4->SR3->SR2-SR1.

The required step is to compute the ratio of shifted sink concentration to the shifted source concentration $\left(\mathrm{Z}_{\mathrm{k}, \mathrm{SKj}} / \mathrm{C}_{\mathrm{k}, \mathrm{SRi}}\right)$ for each sink-source pair and for each contaminant ' $\mathrm{k}$ '. Table 4 shows the computation results. For SK1 and SK3, as all the minimum ratios paired with SR1, SR2, SR3 and SR4 are less than 1, they are impossible to be fulfilled by merely internal sources. This is because the concentrations (either A 
or B) of these sources are all higher than both of the sinks. For SK3, although SR2 has lower concentrations of A and B than SK3, the source flowrate is not enough to cover SK3. So, they require freshwater and should be classified as Below the Pinch in contaminant 'A' Composite Curve. While for SK2 and SK4, the minimum ratios for both sinks to all sources are less than 1, which means they should be classified as sinks that belong to below the Pinch Region.

Table 4: Determination of sink-to-source concentration ratio. The minimum value among the contaminants are highlighted in bold

\begin{tabular}{|c|c|c|c|c|c|c|c|c|}
\hline & $\mathrm{A}\left(\mathrm{Z}_{\mathrm{sk}} / \mathrm{C}_{\mathrm{SR}}\right)$ & $\mathrm{A}\left(\mathrm{Z}_{\mathrm{sk}} / \mathrm{C}_{\mathrm{SR}}\right)$ & $\mathrm{A}\left(\mathrm{Z}_{\mathrm{sk}} / \mathrm{C}_{\mathrm{SR}}\right)$ & $\mathrm{A}\left(\mathrm{Z}_{\mathrm{sk}} / \mathrm{C}_{\mathrm{SR}}\right)$ & & $\mathrm{B}\left(\mathrm{Z}_{\mathrm{sk}} / \mathrm{C}_{\mathrm{SR}}\right)$ & $\mathrm{B}\left(\mathrm{Z}_{\mathrm{sk}} / \mathrm{C}_{\mathrm{SR}}\right)$ & $\mathrm{B}\left(\mathrm{Z}_{\mathrm{sk}} / \mathrm{C}_{\mathrm{SR}}\right)$ \\
\hline & SK1 & SK2 & SK3 & SK4 & & SK1 & SK2 & SK3 \\
\hline SR1 & 0.40 & 1 & 2 & 4 & SR1 & 0.20 & 0.067 & 0.50 \\
\hline $\mathrm{SR} 2$ & 0.20 & 0.50 & 1 & 2 & $\mathrm{SR} 2$ & 0.50 & 0.17 & 1.25 \\
\hline SR3 & 0.13 & 0.33 & 0.67 & 1.33 & SR3 & 0.60 & 0.20 & 1.50 \\
\hline SR4 & 0.08 & 0.20 & 0.40 & 0.80 & SR4 & 0.80 & 0.25 & 1.875 \\
\hline
\end{tabular}

As the total sink flowrates (SK1+SK3) in contaminant cascade ' $A$ ' has the highest flowrate, the design methodology is performed on contaminant cascade 'A' first. The design methodology starts with SK1 first. The source ranking order based on contaminant 'B' is SR1- $>$ SR2- $>$ SR3- $>$ SR4. Figure 7a first presents the source and sink CCs specifically for SK1. Notice that the Pinch occurs at contaminant 'B', with required freshwater as $18.4 \mathrm{t} / \mathrm{h}$.

According to the proposed source allocation methodology in Section 2.4 and 3, since the Pinch does not occur at the sink's main limiting contaminant 'A' - step (i), as well as SR1 and SR2 are conflicting sources, there is still room for freshwater reduction. SR1 allocated to SK1 can be reduced. Using the graphical strategy presented in Section 2.4, SR1 can be reduced until the distance of sink CC to source CC for both contaminants are identical. The source CC is then shifted to the left. Figure $7 \mathrm{~b}$ shows the source and sink CCs with reduced SR1. Otherwise, using the procedure from Section 3, the step (ii) from the source allocation step can be performed. Since this involves two contaminants, pair with two sources from the arranged source, i.e. SR1 and SR2. In step (iii), use Eqs(4-5) to determine the flowrates of SR1 and SR2 to be allocated to SK1. As this is only two contaminants, setting the freshwater requirement- $\mathrm{Eq}(4)$ for A to be equal to B is enough. In step (iv), the flowrates of SR1 and SR2 that can be allocated to SK1 needs to be checked. The determined flowrates are found a: $\mathrm{SR} 1=3.45 \mathrm{t} / \mathrm{h}$ and $\mathrm{SR} 2=2.875 \mathrm{t} / \mathrm{h}$. As both flowrates are not negative and are less than the available flowrates, the step for SK1 is completed. The procedure is then repeated for SK3 - step (vi). It can be noticed that the freshwater requirement is reduced to 16.675 t/h, with only $3.45 \mathrm{t} / \mathrm{h}$ of SR1 to be allocated to SK1- see Figures 9.
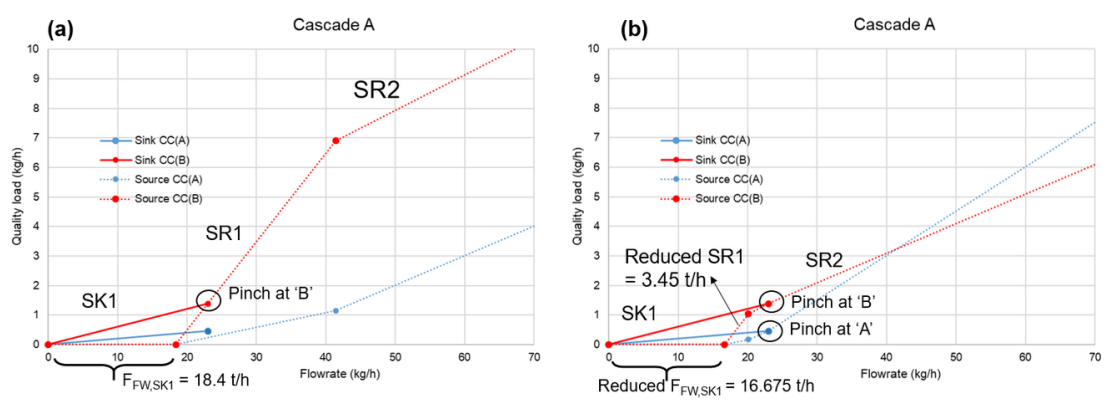

Figure 7: Source and sink CC for SK1 (a) Follow the source ranking order (b) Further freshwater reduction The design methodology is repeated for SK3, and the Composite Curves are stacked above SK1. Figure 8a first presents the source and sink CCs before identifying the freshwater target for SK3. It is worth to 
be noted that contaminant 'B' is not constraining for SK3 if the sources are arranged with ascending order of contaminant 'A'. After shifting the source CC to the right, the Pinch occurs at the sink's main limiting contaminant 'A', see Figure 8b. As the Pinch occurs at the main limiting contaminant, there is no room to further reduce the freshwater target. In this case, the total freshwater target for both SK1 and SK3 is $16.675+13.258=29.933 \mathrm{t} / \mathrm{h}$.
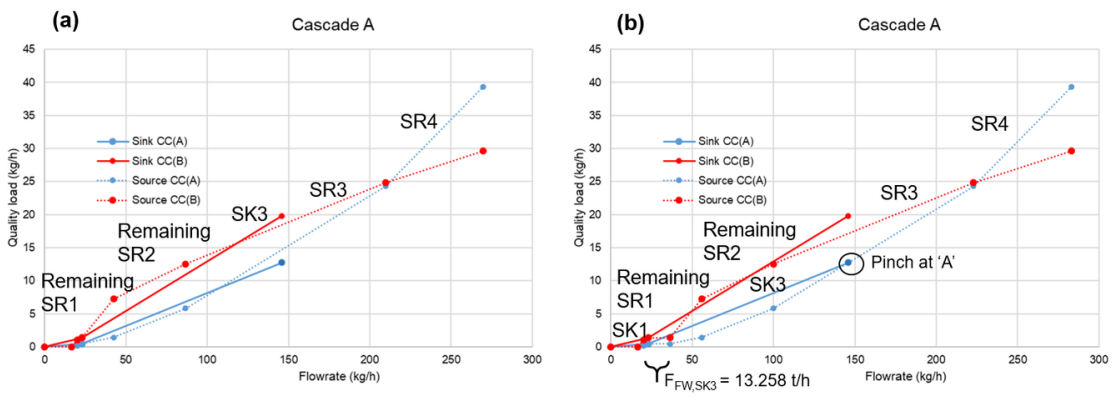

Figure 8: Source and sink CC for SK1+SK3 in contaminant cascade 'A' (a) Before shifting source CC for SK3 (b) After shifting source CC for SK3

The step is repeated again for the sinks that are in contaminant cascade 'B'. The source ranking order based on contaminant 'B' is SR4->SR3->SR2->SR1. The design first starts with SK2. If the sources are allocated based solely on prioritisation of SR4, the Pinch occurs at contaminant 'A' (see Figure 9a). This is not optimal as SR3 can still be allocated due to the limit for contaminant 'B' is not reached. The flowrate of SR4 is then reduced, and SR3 is used until both contaminants have Pinches (see Figure 9b). The identical scenario is observed for SK4 (Figures 11c and d). Notice that in Figure 9d, a triangle is formed around the source and sink CC for contaminant 'A'. This is feasible as the final load of contaminant 'A' for SK4 is still satisfied.

The Composite Curves show exactly the sources to be allocated for each sink. This is because the freshwater target and the Pinch are determined sequentially for each sink. By doing this, the simultaneous target and source allocation for each sink can be obtained. The allocation of sources is directly connected to the network design as well. The detailed illustration of the source allocation to the sinks is shown in Figure 10.

Figure 9: Source and sink CC for scenario 1 case study 1 (a) SK2 (b) SK2 with the reduced freshwater target (c)SK4 (d) SK4 with reduced freshwater target 


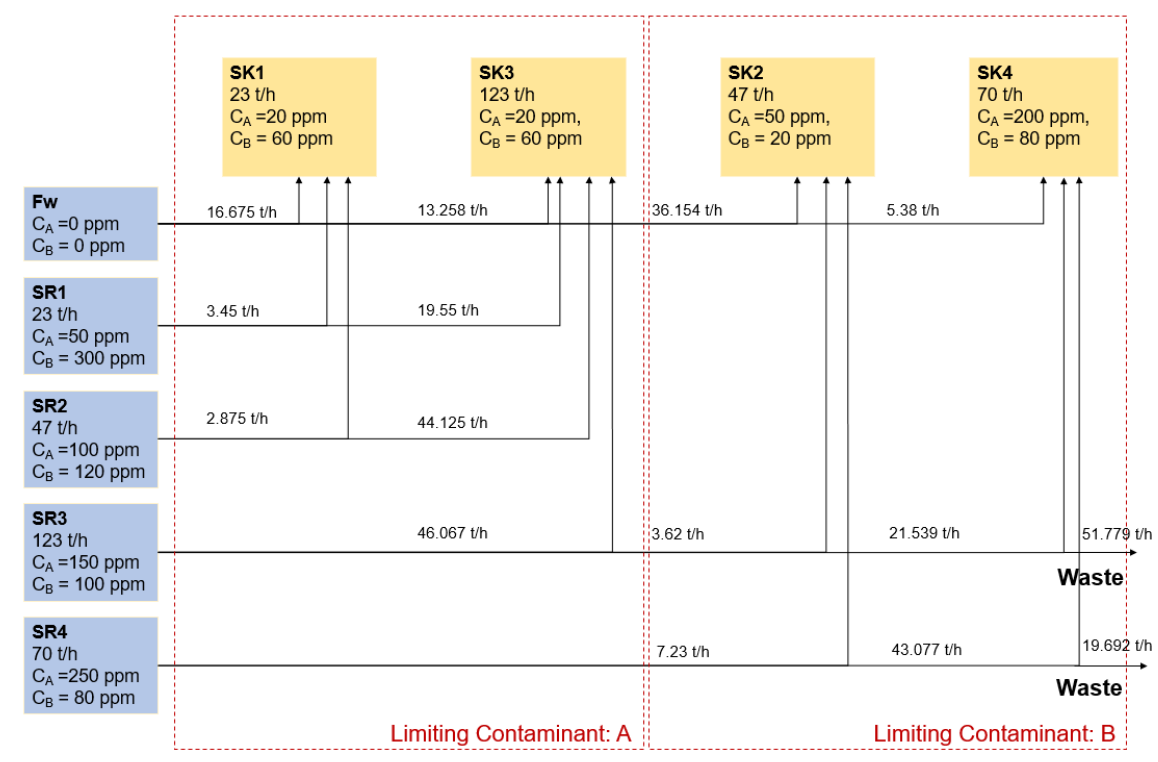

Figure 10: Detailed network design for case study 1

Scenario 2

The source data presented for scenario 1 is shuffled to rearrange the sequence of the sources. The concentrations of sources for contaminant 'B' are rearranged. Table 5 shows the source-sink data. As the maximum source concentrations are unchanged, the ratio of sinks' concentrations to the maximum source concentrations is unchanged as well. This means the sinks, in this case, are still assigned to a similar cascade. All individual sinks-to-source ratios remained below 1 as well, which indicates all the sinks should be located Below the Pinch (require freshwater).

Table 5: Data for scenario 2 of case study 1

\begin{tabular}{llll}
\hline $\mathrm{SR}$ & $\mathrm{F}_{\mathrm{sr}}(\mathrm{kg} / \mathrm{h})$ & $\mathrm{C}_{\mathrm{A}, \mathrm{SR}}(\mathrm{ppm})$ & $\mathrm{C}_{\mathrm{B}, \mathrm{SR}}(\mathrm{ppm})$ \\
\hline 1 & 23 & 50 & 120 \\
2 & 47 & 100 & 80 \\
3 & 123 & 150 & 300 \\
4 & 60 & 250 & 100 \\
$\mathrm{Fw}$ & - & 0 & 0 \\
$\mathrm{SK}$ & $\mathbf{F}_{\mathbf{s k}}(\mathbf{k g} / \mathbf{h})$ & $\mathbf{Z}_{\mathbf{A}, \mathbf{S k}}(\mathbf{p p m})$ & $\mathbf{Z}_{\mathrm{B}, \mathrm{SK}}(\mathbf{p p m})$ \\
1 & 23 & 20 & 60 \\
2 & 47 & 50 & 20 \\
3 & 123 & 100 & 150 \\
4 & 70 & 200 & 80 \\
\hline
\end{tabular}

The design methodology should start with contaminant cascade 'A' first as well. Figures 11 show the plot of the CC for both SK1 and SK3. The source order should be SR1-> SR2 -> SR3 -> SR4. In Figure 11a, the Source CC is shifted to the right until it touches the endpoint of SK1 line. It should be noted that the Pinch occurs at contaminant 'A'. Since SK1 is determined as limited by contaminant 'A', there is no room for fresh resource reduction for SK1- step (i) in Section 3. After SK1 is fulfilled, the next sink to be fulfilled is SK3. Figure 11b shows the plot of the CC with SK3 is stacked above SK1. Notice the Pinch point occurs at contaminant 'B', but this is not the main limiting contaminant for SK3. The flowrate of SR3 should be 
reduced by mixing some of the SR4 to further reduce the freshwater target for SK3. Using the proposed algorithm from Section 2.4/3, the reduced SR3 that can be allocated to SK3 is $42.7917 \mathrm{t} / \mathrm{h}$, with a little from SR4 to reach Pinch points for both contaminants. Figure 11c shows the plot of the CCs.

As for the sinks in contaminant cascade 'B', a similar methodology is applied. The source order is SR2-> SR4- $>$ SR1- $>$ SR3. SK2 is first to be fulfilled. As SR2 and SR1 are allocated to SK1 and SK3, the sources that are remained are SR4 and SR3. By performing PA, the Pinch Points of both contaminants are reached for SK2, by using solely SR4 (see Figure 12a). This is the optimal allocation for SK2. As for the next sink SK4, the Pinch Point also occurs at contaminant 'B- see Figure 12b, which is its main limiting contaminant. There is no room for further freshwater reduction in this case.

Figure 11: Source and sink CC for scenario 2 case study 1 (a) SK1 (b) SK3 (c) SK3 with reduced freshwater target

Figure 12: Source and sink CC for scenario 2 case study 1 (a) SK2 (b) SK4

Scenario 3: Sink Above the Pinch

For this scenario, the concentrations of SK3 are manipulated to higher values to showcase the scenario where the sink is located Above the Pinch. Table 4 shows the data.

Table 4: Data for scenario 3 of case study 1

\begin{tabular}{llll}
\hline $\mathrm{SR}$ & $\mathrm{F}_{\mathrm{sr}}(\mathrm{kg} / \mathrm{h})$ & $\mathrm{C}_{\mathrm{A}, \mathrm{SR}}(\mathrm{ppm})$ & $\mathrm{C}_{\mathrm{B}, \mathrm{SR}}(\mathrm{ppm})$ \\
\hline 1 & 23 & 50 & 120 \\
2 & 47 & 100 & 80 \\
3 & 123 & 150 & 300 \\
4 & 60 & 250 & 100 \\
$\mathrm{Fw}$ & - & 0 & 0 \\
$\mathrm{SK}$ & $\mathbf{F}_{\mathbf{s k}}(\mathbf{k g} / \mathbf{h})$ & $\mathbf{Z}_{\mathbf{A}, \mathbf{S k}}(\mathbf{p p m})$ & $\mathbf{Z}_{\mathrm{B}, \mathrm{SK}}(\mathbf{p p m})$ \\
1 & 23 & 20 & 60 \\
2 & 47 & 50 & 20 \\
3 & 123 & 290 & 300 \\
4 & 70 & 200 & 80 \\
\hline
\end{tabular}

It is worth to note that the concentrations of SK3 for both contaminants are higher than all the concentrations of all the sources. The concentration ratios of SK3 to the maximum sources concentration $\left(\mathrm{Z}_{\mathrm{k}, \mathrm{SK} 3,} / \mathrm{C}_{\mathrm{k}, \mathrm{SR} \text { max }}\right)$ for both contaminants are larger than or equal to $1(290 / 250>1$ and $300 / 300=1)$. This means that all the individual sink-to-source concentration ratios are all larger than 1 . SK3 is not limited by any contaminants, and all the sources can be allocated to SK3. If the sources are enough, then SK3 can be covered without resorting to the freshwater.

The full design methodology has been shown as presented in Scenario 2 and is nearly identical, According to the heuristic presented in Section 2.4/3, SK3 should be the last sink that is fulfilled as it should be located Above the Pinch. In this case, the design methodology should first start with contaminant cascade 'B' as the total flowrates of sinks (SK2+SK4) in contaminant cascade 'B' exceed the flowrate of the sink (SK1) in 
cascade 'A'. After determining the allocation of sources to SK2, SK4 and SK1, the remaining sources can be sent to SK3. Figures 13 show the CC plot for this scenario.

Figure 13: Source and sink CC for scenario 3 case study 1 (a) SK2 (b) SK4 (c) SK1 (d) SK3

\section{Conclusion}

This work has formulated a Pinch-Based simultaneous targeting and synthesis procedure for multicontaminant material recycle-reuse networks, on the example of water networks. It involves the methods to determine the source prioritisation sequence for each sink, classification of sinks to Below/Above Pinch, and determine the limited flowrate of the sources that can be assigned. The allocated sinks and sources are then arranged with ascending order of their concentrations. After the assignment of the sinks and sources to each contaminant cascade, the typical PA can then be performed for each contaminant cascade and for each sink sequentially. If the Pinch Point occurs at the non-limiting contaminant of the sink, a source reduction can be performed to further reduce the freshwater target for that sink. The proposed method is validated with an illustrative example and two real case studies from the paper mill and starch industry. The obtained freshwater targets for both cases studies are $39,836 \mathrm{kt} / \mathrm{d}$ and $47.36 \mathrm{~kg} / \mathrm{h}$. The results agree well with the solution from the literature and the solutions obtained by solving the superstructure model. The consistent solutions obtained compared with optimisation method and previous works prove the accuracy and legitimacy of the method.

Note that the freshwater requirements determined in this work represent the resource requirement ignoring the interaction between the sinks and sources are. The data used in this work are the maximum flowrates and concentrations of the sources and sinks. Certain sink's maximum concentration limit might not be reached, and this could affect the concentration of the sources from the same operation of the sink. If fixed contaminant load is assumed for each unit operation, the concentration of the source might be reduced as well, and the overall fresh requirement can be reduced. For fixed load operation, the flowrates of the sink and source can be reduced, as long as the fixed mass load is guaranteed. This could lead to a reduction in a fresh resource. However, future research should incorporate the realistic relationships between the inlet and outlet contaminant concentrations. The interaction between multiple contaminants in either inlet or outlet streams should be taken into account as well. Future research can be focused on a Multi-Contaminant Cascade Table Analysis. It can be developed for automated resource targeting. Water regeneration potential and multiple fresh resources with prioritised objectives should be incorporated into the analysis as well.

\section{Acknowledgement}

The funding from the project 'Sustainable Process Integration Laboratory - SPIL funded by EU' CZ Operational Programme Research and Development, Education, Priority1: Strengthening capacity for quality research (Grant No. CZ.02.1.01/0.0/0.0/15_003/0000456) under the collaboration agreement with Universiti Teknologi Malaysia has been gratefully acknowledged.

\section{References}

1. Fan YV, Lee CT, Lim JS, Klemeš JJ, Le PTK. Cross-disciplinary Approaches Towards Smart, Resilient and Sustainable Circular Economy. Journal of Cleaner Production. 2019;232: 1482-1491.

2. El-Halwagi MM, Manousiouthakis V. Synthesis of Mass Exchange Networks. AIChE Journal. 1989;35(8):1233-1244

3. Linnhoff B, Townsend DW, Boland D, Thomas BEA, Guy AR, Marsland RH. A user guide on process integration for the efficient use of energy (2nd ed). Rugby, UK: IChemE. 1982.

4. Wang YP, Smith R. Wastewater minimisation. Chemical Engineering Science.1994; 49:981-1006. 
5. Olesen SG, Polley GT. A Simple Methodology for the Design of Water Networks Handling Single Contaminants. Chemical Engineering Research and Design. 1997; 75:420-426.

6. Hallale N. A new graphical targeting method for water minimisation. Advances in Environmental Research. 2002;6:377-390.

7. Gomes JFS, Queiroz EM, Pessoa FLP. Design procedure for water/wastewater minimisation: single contaminant. Journal of Cleaner Production. 2007;15:474-485.

8. El-Halwagi MM (Ed.). Synthesis of mass exchange networks: A graphical approach, In: Process Systems Engineering, Process Integration. Massachusetts: Academic Press, 2006:87-136.

9. El-Halwagi MM, Gabriel F, Harell D. Rigorous Graphical Targeting for Resource Conservation via Material Recycle/Reuse Networks. Industrial \& Engineering Chemistry Research. 2003; 42: 43194328.

10. Foo DCY. State-of-the-Art Review of Pinch Analysis Techniques for Water Network Synthesis. Industrial \& Engineering Chemistry Research. 2009;48:5125-5159.

11. Klemeš JJ, Kravanja Z. Forty years of Heat Integration: Pinch Analysis (PA) and Mathematical Programming (MP). Current Opinion in Chemical Engineering. 2013; 2(4): 461-474.

12. Klemeš JJ, Varbanov PS, Walmsley TG, Jia X. New directions in the implementation of Pinch Methodology (PM). Renewable and Sustainable Energy Reviews. 2018;98: 439-468.

13. Alva-Argáez A, Vallianatos A, Kokossis A. A multi-contaminant transhipment model for mass exchange networks and wastewater minimisation problems. Computers \& Chemical Engineering. 1999;23:14391453.

14. Ulson de Souza AA, Forgiarini E, Brandão HL, Xavier MF, Pessoa FLP, Souza SMAGU. Application of Water Source Diagram (WSD) method for the reduction of water consumption in petroleum refineries. Resources, Conservation and Recycling. 2009;53(3):149-154.

15. Karthick R, Kumaraprasad G, Sruti B. Hybrid optimisation approach for water allocation and mass exchange network. Resources, Conservation and Recycling. 2010;54(11):783-792.

16. Gomes JFP, Mirre RC, Delgado BEPC, Queiroz EM, Pessoa FLP. Water sources diagram in multiple contaminant processes: maximum reuse. Industrial Engineering and Chemistry Research. 2013;53(4):1667-1677.

17. Calixto EES, Francisco FS , Pessoa FLP, Queiroz EM. A Novel Approach to Predict Violations and to Define the Reference Contaminant and Operation in Water Using Networks. Computer Aided Chemical Engineering. 2015;37: 1901-1906.

18. Francisco FS, Mirre RC, Calixto EES, Pessoa FLP, Queiroz EM. Water sources diagram method in systems with multiple contaminants in fixed flowrate and fixed load processes. 2018;172:3186-3200

19. Calixto EES, Pessoa FLP, Mirre RC, Francisco FS ,Queiroz EM. Water Sources Diagram and Its Applications. 2020;8(3):313

20. Castaño JA, Higuita JC. Using turbidity for designing water networks. Journal of Environmental Management. 2016;172:129-135.

21. Mabitla SS, Majozi T. A hybrid method for synthesis of integrated water and regeneration networks with variable removal ratios. Journal of Environmental Management. 2019;231:666-678.

22. Liu ZY, Yang Y, Wan LZ, Wang X, Hou KH. A heuristic design procedure for water-using networks with multiple contaminants. AIChE Journal. 2009;55: 374-382.

23. Fan XY, Li YP, Liu ZY, Pan CH. A new design method for water-using networks of multiple contaminants with the concentration potential concepts. Chemical Engineering Science. 2012;73: 345-353.

24. Li AH, Fan XY, Klemeš JJ, Liu ZY. Concentration potential concepts: Powerful tools for design of water-using networks with multiple contaminants. Journal of Cleaner Production. 2017;165: 254-261.

25. Zhao HP, Yang Y, Liu ZY. Design of heat integrated water networks with multiple contaminants. Journal of Cleaner Production. 2019;211: 530-536.

26. Wang YP, Smith R. Wastewater minimisation with flowrate constraints. Transactions of the Institute of Chemical Engineers. 1995;73: 889-904.

27. Chin HH, Liew PY, Varbanov PS, Klemeš JJ. Pinch-based Targeting Methodology for Multi-Contaminant Material Recycle/Reuse. Chemical Engineering Science. 2020. DOI: 
10.1016/j.ces.2020.116129

28. Short M, Isafiade AJ, Biegler LT, Kravanja Z. Synthesis of mass exchanger networks in a two-step hybrid optimisation strategy. Chemical Engineering Science. 2018; 178:118-135.

29. Oladosu WA, Wan ALwi SR, Manan ZA. A new algebraic tool for simultaneous targeting and design of a mass exchange network with stream splitting for sustainable environment. Journal of Cleaner Production. 2020;249: 119361.

30. Yanwarizal, Oladosu WA, Wan Alwi SR, Manan ZA, Fraser D. A new graphical approach for simultaneous targeting and design of mass exchange networks. Computers \& Chemical Engineering. 2020;142: 107061.

31. Yang M, Feng X, Chu KH, Liu G. Graphical method for identifying the optimal purification process of hydrogen systems. Energy. 2014;73: 829-837.

32. Teles J, Castro PM, Novais AQ. LP-based solution strategies for the optimal design of industrial water networks with multiple contaminants. Chemical Engineering Science. 2008;63(2): 376-394.

33. Lovelady EM, El-Halwagi MM, Krishnagopalan GA. An integrated approach to the optimisation of water usage and discharge in pulp and paper plants. IJEP. 2007;29: 274.

34. Dakwala M, Mohanty B, Bhargava R. A process integration approach to industrial water conservation: a case study for an Indian starch industry. Journal of Cleaner Production. 2009;17: 1654-1662. 